Proceedings of the 2010 Winter Simulation Conference

B. Johansson, S. Jain, J. Montoya-Torres, J. Hugan, and E. Yücesan, eds.

\title{
SPATIAL MODELING IN CELL BIOLOGY AT MULTIPLE LEVELS
}

\author{
Arne T. Bittig \\ Adelinde M. Uhrmacher \\ University of Rostock \\ Albert-Einstein-Str. 21 \\ Rostock, 18059, GERMANY
}

\begin{abstract}
Most modeling and simulation approaches applied in cell biology assume a homogeneous distribution of particles in space, although experimental studies reveal the importance of space to understand the dynamics of cells. There are already numerous spatial approaches focusing on the simulation of cells. Recently, they have been complemented by a set of spatial modeling languages whose operational semantics are tied partly to existing simulation algorithms. These modeling languages allow an explicit description of spatial phenomena, and facilitate analysis of the temporal spatial dynamics of cells by a clear separation between model, semantics, and simulator. With the supported level of abstraction, each of those offers a different perception of the spatial phenomena under study. In this paper, we give an overview of existing modeling formalisms and discuss some ways of combining approaches to tackle the problem the computational costs induced by spatial dynamics.
\end{abstract}

\section{INTRODUCTION}

Based on advanced experimental biological methods, e.g. high content screening or high resolution microscopy, recent findings emphasize the central role that space plays in inter- and intracellular dynamics (Kholodenko 2006). Already the functioning of individual signaling pathways depends on the location and movement of their key players, the more so if the crosstalk between pathways (Dumont, Pécasse, and Maenhaut 2001, Bardwell et al. 2007) shall be analyzed, i.e. situations where proteins might belong to more than one pathway and thus play a crucial role in mediating between individual pathways and full intracellular interaction networks. Generally, the more realistic models become, the more urgent is the need to take the relay of information in and between cells, depending on diffusion and active transport, into consideration.

Takahashi, Arjunan, and Tomita (2005)'s seminal paper on space in systems biology discussed a set of approaches with origins from physics to computer science offering different perceptions on the system under study. However, in this discussion a clear separation of concerns (Dijkstra 1982), i.e. modeling languages that allow a declarative specification combined with a clear operational semantics that can give rise to multiple simulation engine implementations on the one hand and efficient simulation and analysis engines on the other hand, was of less importance. Since then, approaches have been developed addressing specific concerns, e.g. more efficient spatial simulation algorithms, or modeling formalisms. The latter shall be the focus of this paper. As modeling means structuring the knowledge about a given system taking the objective of the simulation study into account, the question arises what expressiveness a language should exhibit and what level of abstraction it supports.

In the following section we will first try to clarify the terms level and scale, as both will play a role in categorizing the different approaches toward spatial dynamics. After that, we will give an overview of space in modeling and simulation in systems biology based on Takahashi, Arjunan, and Tomita (2005). In section four we discuss different spatial modeling formalisms and relate them to the just discussed approaches. As modeling formalisms offer specific perspectives, we will discuss the combination of those and the promise these combinations hold for keeping the complexity of spatial modeling and simulation at bay. 


\section{LEVELS, SCALES, AND RESOLUTION IN SPATIAL MODELING}

The terms level, scale, and resolution refer to certain aspects of models or the modeled phenomena and their use varies from research field to field. While multi-level, multi-scale, and multi-resolution are sometimes used synonymously (Weinan and Engquist 2003, Brandt 2001, Dolbow, Khaleel, and Mitchell 2004), this is not always the case and especially in the context of this paper we would like to distinguish between them.

We use level to refer to levels of abstractions, e.g. in terms of molecular or cellular dynamics, or to cellular automata, lattice based reaction and diffusion equations etc. Thus, multi-level modeling refers to the area of application as well as to introducing abstraction levels due to modeling. In the former case multi-level modeling will reflect the structure of the knowledge in the domain, in the latter case modeling itself will introduce additional structures, e.g. to handle the complexity of the system. This interpretation of the term is in line with its use in organizational research, social sciences, and the statistics where multi-level refers to study interacting processes taking place at different organizational levels of society (Tilly 1997), or to the modeling and simulation of a society at individual and population level (Möhring 1996). Similarly, consider a biological system: relevant levels of abstractions may include cell populations, individual cells, cell organelles, pathways, down to individual molecules inside the cell or treating part of the system at concentration level and other part of the system as individual molecules (Degenring, Röhl, and Uhrmacher 2004). In the ideal case we will find that the model structure also reflects the different abstractions of the application area. However, a multi-level model in cell biology might also be an ODE model that refers to dynamics at inter- and intra-cellular levels (Lai et al. 2009). Everything is encoded at the same level of abstraction referring to modeling, i.e. as a set of variables and differential equations. As modeling is concerned with abstraction, multi-level modeling touches at the core of modeling and simulation.

Multi-scale modeling or simulation refers to a scale in, e.g. size, numbers, time, or space and requires to take widely spread areas of this scale in the phenomena being studied into account, for example both small inorganic molecules and large protein complexes playing a role, processes working on nano-seconds and hours, or some molecules available in vast abundance whereas others are rare. Obviously, scales are a straight-forward way to categorize similar things. $\mathrm{Li}$ et al. (2005), in turn, propose to use the term level for categorization along layers in a hierarchy (cf. figure 1 and scales only for subdivisions within one level. However, they themselves are not entirely consistent in their use of the terms, and this is usage is far from universal. In cell biology, multi-scale simulation typically refers to different spatial extensions or, in the appropriate context, different time scales (Takahashi et al. 2004).

Whereas multi-level and multi-scale refer primarily to the relation between modeling and system to be modeled, multi-resolution refers to the relation between simulation and model and thus is of less relevance here.

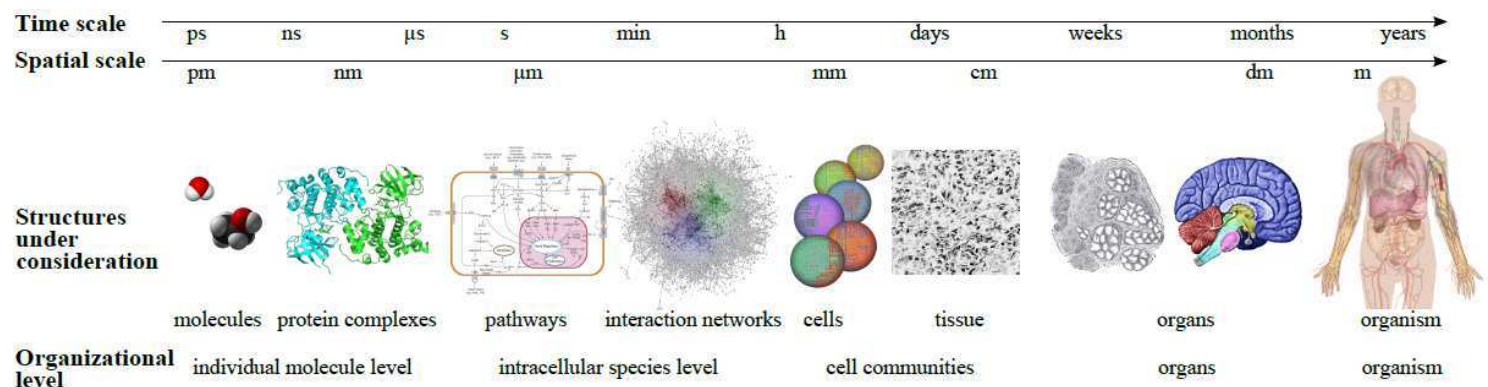

Figure 1: Structures relevant in biology, their spatial extension and the time scale they are often considered at, illustrating the ambiguity in the terms level and scale in systems biology. Li et al. (2005) (from whose figure 1 the inspiration for this one was taken) consider micromolecules and macromolecule complexes different scales within the "molecule" organizational level. In analogy, molecule interactions, pathways, and the full interaction network could be different scales within the intracellular level, but they can also be considered different levels of abstraction.

\section{REPRESENTATION OF SPACE IN MODELING AND SIMULATION}

Also non-spatial modeling formalism can easily cover certain spatial features. For example, even if there is no explicit way to define spatial structures like compartments, instead of defining a species (or, more general, to-be-simulated entity) $A$, one can instead use two entities AInCompartment1 and AInCompartment2 and define what would otherwise be transport of $A$ between compartments as conversion between species. In the original Bio-PEPA (Ciocchetta and Hillston 2008), a process-algebra-based modeling formalism, for example, this is an explicitly intended way to represent compartments. This approach can easily be adopted by other approaches, e.g. ODEs (Lai et al. 2009). However, it makes modeling spatial phenomena cumbersome due to the combinatorial explosion associated with the need to specify each reaction 


\section{Bittig and Uhrmacher}

for each compartment, the representation of transport being not yet included. If merging and changing volume of compartments is added, this seems hardly feasible. Additionally, in this approach the compartments are only implicitly represented by the variable names. In the following, we will focus on modeling formalisms that support an explicit notion of space. But first we will identify crucial criteria in representing space by discussing spatial approaches as presented by Takahashi, Arjunan, and Tomita (2005).

compartmental: If one species has different reaction partners in different compartments of a cell, it is necessary to know its concentrations in each compartment for proper representation of its reaction dynamics. For example, the transcription factor protein Mig1 represses some genes when unphosphorylated, but it moves out of the nucleus when phosphorylated (which may happen inside the nucleus). It can thus get dephosphorylated again only outside the nucleus, and in order to function as a transcriptional repressor again will obviously have to move back inside the nucleus (Ahuatzi et al. 2007). Dynamics inside one compartment are typically realized using a non-spatial modeling approach. This most basic, qualitative approach to incorporating (discrete) spatial features does not need to represent actual molecule positions. Compartments may be nested, i.e. each compartment may contain one or more sub-compartments (as depicted in figure 2 (a)), however, the simple distinction of, for example, nucleus and cytosol would also qualify here. Typically, the compartmental approach of representing space is used at the level of pathways and interaction networks up to cells and tissues. They are less common for molecules and protein complexes, although examples do exist (Maus 2008).

continuous - particle space: For most realistic modeling of molecular motions in space, each particle is represented individually using rules arising from fundamental physics with coordinates and a trajectory in a continuous space. A manybody problem with only computationally expensive solutions or approximations arises. The most fundamental approach, molecular dynamics (MD; Adcock and McCammon 2006; figure 2 (e)), tackles this by numerically solving Newton's laws, which is feasible only for very small scales both in time and space, as computation effort required is proportional to the number of interacting particles. Dissipative particle dynamics (DPD) is a coarse-grained approximation of MD (Heyes et al. 2004), but does not permit biochemical interactions and is thus not suitable for simulation of biochemical systems. A non-deterministic approach, i.e. one representing random noise in particle movements, is that of Brownian dynamics, relying on the Langevin equation (Ermak and McCammon 1978, Northrup, Allison, and McCammon 1984). Like in molecular dynamics, a continuous differential equation system has to be solved numerically, and it is also computationally expensive, especially the more crowded the environment is. Assuming that, when choosing time steps of sufficiently small size, no more than two molecules react at the same time, the many-body problem can be reduced to two-body problems, for which an analytical solution exists. Green's function reaction dynamics (van Zon and ten Wolde 2005; figure 2 (f)) thus approximates the solution of Brownian dynamics, turning it into a discrete event type problem. These computations are faster especially when distances between molecules are not too small (allowing larger time steps).

continuous - gradients/PDEs: Whereas ordinary differential equations describe time-dependency of one variable (in systems biology usually species concentration) on other variables of the same type, partial differential equations (PDEs) allow description of dependency on more than one variable, e.g. spatial position besides time. However, individual particles are not represented here, only concentration gradients across space (figure 2 (d)). This has been applied to microbiological phenomena like the behavior of receptors on a membrane (Haugh 2002), and via reaction-diffusion equations also to whole cells (Kholodenko 2006). Numerical integration of the PDEs requires generation of a mesh - the finer the mesh (Slepchenko et al. 2003), the more accurate the approximation, but the higher the computational costs. PDE-based simulations are fully deterministic and thus cannot cover stochastic effects from random noise. In addition, like all purely continuous approaches, they are badly equipped for handling discontinuity.

discrete - lattice: Whereas the previous approaches represent spatial aspects by real-valued coordinates, space can also discretized by splitting it into regions considered separately (figure 2 (b)), i.e. projecting a lattice onto it with certain assumptions about each lattice element. Cellular automata (Alber et al. 2002) are the most prominent representative of this group. They consist of a lattice of appropriately small uniform blocks, each of which may be occupied by one or no molecule. In discrete time steps, movement of particles is realized as propagation to a nearby block in the lattice, thus one block's state depends only on the previous state of its neighbors and itself. When two particles are to move into the same block, special treatment of a collision or reaction type event is triggered. This has the potential to drastically reduce computational complexity, but requires the block size to reflect average molecule distance and average molecule diameter. The approach is being applied mostly for studying cell populations and specific formations of tissue. It moves small fractions of space itself into the focus of interest, being or being not occupied by a key player of cellular dynamics. This micro-scale approach thus does not cope well with differences in molecule numbers and sizes (i.e., multiple scales).

discrete - subvolume: The aforementioned problem of coping with varying numbers can be overcome by using a more coarse-grained lattice and allowing several molecules in each lattice element, i.e. in a subvolume (figure 2 (c)). This can be done, for example, by appropriate adaptations of cellular automata (Shimizu, Aksenov, and Bray 2003, Stundzia and Lumsden 1996), or by extending Gillespie's method (Gillespie 1976) spatially (Elf, Doncic, and Ehrenberg 2003), considering the molecules to be distributed uniformly and moving and reacting according to Gillespie's algorithm, but 
(a) compartments

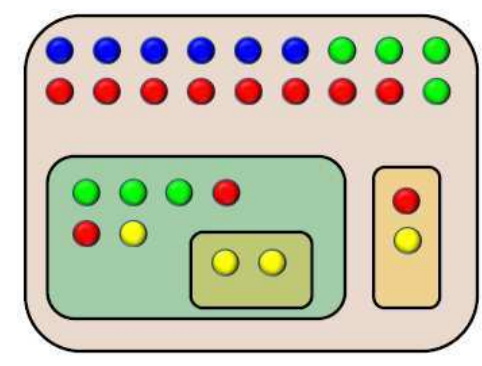

(d) continuous - gradients

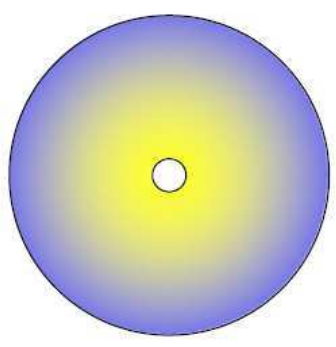

(b) discrete - lattice (grid)

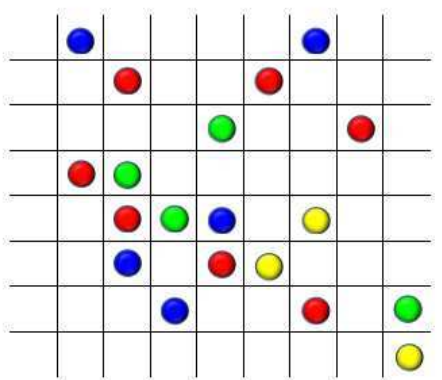

(e) continuous - particles

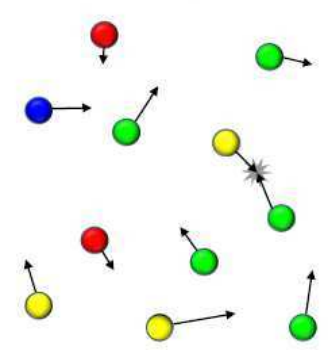

(c) discrete - subvolumes

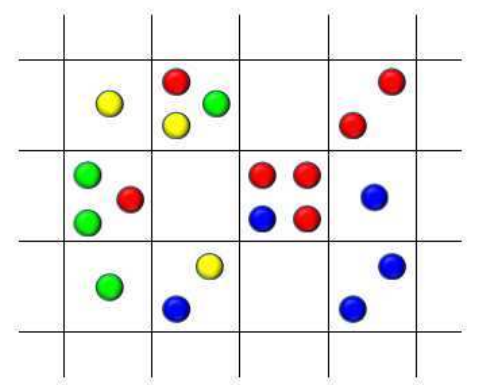

(f) continuous - particles

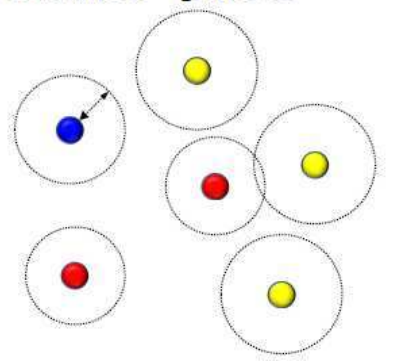

Figure 2: Different ways of representing space in a model or a simulation (adapted from Takahashi, Arjunan, and Tomita 2005, figure 1). (a) molecules/species can be in different compartments and interactions happen only within one compartment, apart from transport between compartments that has to be modeled explicitly; (b/c) space is divided into equal blocks of a defined neighborhood relation, usually a lattice of cubes, either (b) small cubes occupied by no more than one particle or (c) subvolumes taking several (up to arbitrary many); (d) model describes not particles, but concentrations and their gradients across space via appropriate boundary conditions; (e/f) particles are associated with position and motion in space, collisions between particles trigger reactions. In models close to the laws of physics (e), even particles farther apart influence each other's movement to a small extent. Computational costs are lowered when neglecting influences on a particle beyond a certain distance (sphere of influence) (f), without sacrificing accuracy when ensuring that time steps are sufficiently small that particles cannot travel beyond them.

also occasionally diffusing into neighboring subvolumes (when the movement function's target is outside the current one). Whereas varying numbers are no problem for this approach, varying spatial dimensions are, as the approach does not consider that individual molecules might occupy space.

These major approaches to spatial simulation were developed with different applications in mind and offer different granularity in the approximation of the physical processes. From the computationally very expensive molecular dynamics to the rather elementary discrete grid of cellular automata that allows much faster simulation, the main tradeoff is between accuracy and calculation time, making each approach more or less fitting for different types of phenomena. The PDE approach sticks out as it does not cover individual particles but rather distribution gradients, the purely compartmental approach deviates from the others in that it does not describe motion in the continuous space or a discretization thereof, but only movements between a finite number of compartments.

\section{MODELING FORMALISMS AND THEIR SEMANTICS}

\subsection{Differential Equations}

With differential equations, there is no clear separation between formalism and interpretation, as the term comprises both a well-defined way to specify the equations (syntax) and their mathematical meaning (semantics). Simply put, the equations describe changes in variables representing species concentrations over time depending on other variables representing other species' concentrations. Since abstractions regarding the reaction dynamics have to be made when modeling on the population level, the reaction kinetics, e.g. mass action, Michaelis-Menten or detailed enzyme kinetics, are thus incorporated into the equations' structure, i.e. the formal part. Some software tools supporting modeling and simulation of biological systems using ordinary differential equations (ODEs), e.g. SBtoolbox2 (Schmidt and Jirstrand 2006), have a a text-based input format based on equations. 
Table 1: Modeling formalisms and their capabilities regarding spatial modeling. Letters in column headings refer to the panels of figure 2. Other columns: dynamic cell structures: ability to change/merge/create compartments at run time, e.g. for describing a virus docking to a cell and releasing its content into the host cell. individual-/population-based: entities described in the model are particles of different species, or whole species themselves, respectively (not considering possible singleton species in rule-based models). category: type of modeling formalism (Zeigler, Kim, and Praehofer 2000). Entries: o: explicitly supported by formalism. -: not supported. *: supported with limitations, or in a specially adapted variant of the formalism, or only implicitly, see text

\begin{tabular}{|c|c|c|c|c|c|c|c|}
\hline $\begin{array}{l}\text { modeling formal- } \\
\text { ism }\end{array}$ & $\begin{array}{l}\text { explicit } \\
\text { compart- } \\
\text { ments } \\
\text { (a) }\end{array}$ & $\begin{array}{l}\text { dynamic } \\
\text { cell } \\
\text { struc- } \\
\text { tures }\end{array}$ & $\begin{array}{l}\text { contin- } \\
\text { uous } \\
\text { motion } \\
(\mathrm{d}-\mathrm{f}) \\
\end{array}$ & $\begin{array}{l}\text { lattice- } \\
\text { based } \\
\text { space } \\
\text { (b/c) } \\
\end{array}$ & $\begin{array}{l}\text { indivi- } \\
\text { dual- } \\
\text { based }\end{array}$ & $\begin{array}{l}\text { popula- } \\
\text { tion- } \\
\text { based }\end{array}$ & category \\
\hline Cellular Automata & - & - & - & $\overline{\mathrm{O}}$ & $\overline{\mathrm{O}}$ & - & discrete step-wise \\
\hline DCA & - & $*$ & $*$ & $\mathrm{O}$ & $\mathrm{o}$ & - & discrete-event \\
\hline BioAmbients & o & o & - & - & o & - & discrete-event \\
\hline Bio-PEPA & $*$ & - & - & - & - & o & discrete-event/cont. \\
\hline cBNGL & $\mathrm{O}$ & - & - & - & - & $\mathrm{O}$ & discrete-event \\
\hline LBS & o & - & - & - & - & o & discrete-event/cont. \\
\hline $\mathcal{L} \pi$ & o & - & - & o & o & - & discrete-event/cont. \\
\hline Attributed Pi & $\mathrm{O}$ & $\mathrm{O}$ & - & $*$ & o & $\mathrm{o}$ & discrete-event \\
\hline Space Pi & - & $*$ & $\mathrm{O}$ & - & $\mathrm{o}$ & - & hybrid \\
\hline Shape Calculus & - & $*$ & o & - & $\mathrm{o}$ & - & hybrid \\
\hline PDEs & - & - & $*$ & - & - & $\mathrm{O}$ & continuous \\
\hline
\end{tabular}

There are other modeling formalisms with ODE semantics, i.e. while the model is specified in some other manner, the respective simulation amounts to ODE integration again. Sometimes these semantics are the results of adaptation of an existing modeling formalism to the continuous realm, e.g. continuous petri nets (Recalde, Haddad, and Silva 2007), sometimes they are just alternatives and approximations of other, often stochastic semantics, e.g. the stochastic $\pi$ calculus (Stefanek, Vigliotti, and Bradley 2009) (although it is debatable whether one formalism can have different semantics or whether with a change of semantics, a new formalism is created), some (covered in the next section) were created specifically to allow easier or more general description of reaction rules.

For spatial modeling, ODEs describing species concentration change depending on other species concentrations are extended to partial differential equations (PDEs) also involving spatial degrees of freedom. These represent gradients in the intracellular or intracompartmental distribution of each species, e.g. when a kinase localizes to the cell membrane, but the respective phosphatase is distributed homogeneously (Kholodenko 2006). Instead of global initial concentrations of species, initial distributions and boundary conditions have to be given in advance. The numerical solution of these reaction-diffusion equations relies on a grid of spatial elements used for the approximation (Schaff et al. 1997). So while space is represented as continuous in the PDEs, the simulator will consider discrete points in space, but invisible to the user and not in the same sense as with cellular automata or methods with explicit subvolumes.

The Virtual Cell modeling environment is a tool allowing the specification of models involving compartments of specific shape and the simulation of these models using also partial differential equations (Slepchenko et al. 2003, Yang and Iglesias 2009), although modeling has to be done using the provided graphical user interface and not through a specified formalism. This also applies to other software tools allowing the specification of compartments as well as (ordinary) differential equation-based simulation, e.g. COPASI (Hoops et al. 2006) or CellDesigner (Funahashi et al. 2008).

\subsection{Process Algebras}

In computer science, process calculi have long been used for modeling concurrent systems formally. They provide syntactic constructs to describe processes, usually computations performed on some device, and algebraic rules (hence the synonymous term process algebras) allowing formal reasoning about the descriptions. Since close to a decade they are now being applied to biological systems. Some variants include some form of spatial representation, e.g. the ambient calculus for modeling concurrent processes on mobile devices organized in a network of variable topology (Cardelli and Gordon 2000), others have been adapted to take spatial properties or other special features of biological systems into account.

They can roughly be grouped into two categories: The molecules-as-computation approach, where model entities are individual particles or species described in terms of their interaction capabilities, and population-based approaches 


\section{Bittig and Uhrmacher}

often relying on rule-based descriptions of reactions. Individual-based models may still be simulated by lumping those of one species together.

The $\pi$-calculus (Milner 1999) and specifically its stochastic extension (Priami 1995) serve as the basis for many of the former. While the origin of process algebras is the analysis of concurrent processes (e.g. running on a computer) communicating with each other, reacting particles can also be described (Regev and Shapiro 2004). Thus, particles of one species are considered processes of the same kind running in parallel (an initial configuration of processes, i.e. initial amounts of each species, has to be given). Reactions consist, broadly speaking, of processes sending or receiving messages via dedicated channels, indicating, respectively, willingness to react or availability of a reaction partner, and continuing as certain different processes after successful communication (i.e. a reaction). Spatial extensions of these process algebras were mostly devised with compartments in mind, only few deal with actual coordinates assigned to processes.

Process algebras that cover compartmentalization, nested or not, but no further spatial attributes, and thus assume homogeneous distribution of species within a compartment, include BioAmbients (Regev et al. 2004), Beta Binders (Priami and Quaglia 2005), Brane Calculi (Cardelli 2005), and other variants of the $\pi$ calculus (John et al. 2009). (It has been shown that attributed $\pi$, discussed later, can express these approaches.) Beta Binders, however, relying on boxes with interaction capabilities encapsulating $\pi$ processes, are aimed at describing binding of molecules and do not allow explicit exchange of components between boxes and thus not particularly suitable to model actual cellular compartments. Brane Calculi were designed to model dynamic structures arising from changing membrane configurations and do not include any kind of objects inside the compartments (other than maybe further, nested compartments), and therefore are not quite what we are looking for either. BioAmbients, then, constitute a formalism designed explicitly for spatial modeling. They introduce the notion of the ambient as a "bounded place where computation happens" that can merge with and move inside other ambients or be expelled from them. Ambients are thus not only abstractions for compartments, but also, e.g., protein complexes. Actual computations are defined in a language similar to the $\pi$ calculus. Rates may be associated with reactions, which is possible for example in appropriate extensions of Beta Binders (Degano et al. 2006) and BioAmbients (Brodo, Degano, and Priami 2007) as well as the aforementioned formalisms based on the stochastic $\pi$ calculus (Mazemondet et al. 2009), describing probabilities of possible communication events. Ideally, the semantics are thus those of continuous-time Markov chains (CTMCs), and the models can be simulated with some variant of Gillespie's algorithm.

Bio-PEPA (Ciocchetta and Hillston 2008) is a process-algebra based modeling language for which a variant with explicit compartments is also available (Ciocchetta and Guerriero 2009). It follows, in its authors' words, a "processes as species and not processes as molecules" abstraction, i.e. the model entities are abstractions of molecule species and the model describes changes in the amount of each species. Reactions are still not represented explicitly, only by shared channel names. Aside from stochastic simulation it is also possible to convert species amounts to concentrations and derive ODEs approximating the stochastic solutions (Galpin 2010).

Rule-based modeling languages, in turn, do not focus on the species themselves, but rather give explicit rules regarding conversion between species (Hlavacek et al. 2006). Some, e.g. $\kappa$ (also known as the $\kappa$-calculus, Danos et al. 2007, Danos et al. 2009), were designed to address combinatorial explosion associated with many different variants of a protein being able to participate in reactions with another protein of which also many different subtypes exist, e.g. MAP-kinases and MAPKKs, and deal with this by establishing generic and concrete agents and rules inspired by object-oriented programming. Approaches that allow representation of at least compartmental space include cBNGL and the Language for Biological Systems (LBS).

BioNetGen (Blinov et al. 2004) was originally a software tool for rule-based description of reaction systems that were ultimately translated into ODEs. It has since evolved to include graphical representations and a modeling language closely related to $\kappa$. The latter's latest incarnation, the compartmental BioNetGen language (cBNGL; Harris, Hogg, and Faeder 2009), includes a static topology of compartments and allows both universal (global) reaction rules as well as scope-restricted ones (local to a compartment) while adding transport rules.

LBS (Pedersen and Plotkin 2010) is a recently introduced powerful, modular language that is also semantically flexible, allowing translation of its programs into Petri nets, ODEs, and CTMCs. There is also a compiler from LBS to the Systems Biology Markup Language (SBML; Hucka et al. 2003), a format that is understood by a multitude of simulation tools in cell biology (including those mentioned in the previous section on ODEs).

In a spatial extension of the $\pi$ calculus (which also has alternative deterministic semantics, i.e. can be translated to ODEs), simply called $\mathcal{L} \pi$ (Stefanek, Vigliotti, and Bradley 2009), compartments are arranged according to a given location graph describing the processes' movement possibilities, thus allowing nesting as well as arranging of compartments in a grid or lattice and requiring definition of a movement function for every edge in the graph. (We here prefer the term lattice to grid to avoid confusion of lattice-based modeling of space with the grid-based simulation from parallel and distributed computing.) The space of interest can thus be partitioned into a lattice akin to as it is done in the Next Subvolume Method (NSM, Elf and Ehrenberg 2004), an approximation of Gillespie's algorithm in space.

The attributed $\pi$ calculus $\pi(\mathcal{L})$ with priorities (John et al. 2010) also allows definition of an arbitrarily large (but finite) number of parameterized compartments. The parameters allow establishing a neighborhood relation and facilitate definition of a function for movement between neighbors, although these attributes can be of any type and at the 


\section{Bittig and Uhrmacher}

simulation level it is not known whether they actually represent coordinates. They allow description of a lattice as the aforementioned approach relating to the Next Subvolume Method, but in contrast the separation of reaction and diffusion type events does not come naturally in $\pi(\mathcal{L})$. In addition to the processes as molecules view common with the $\pi$ calculus, here a processes as reactions approach is also possible, i.e. processes change attributes representing species amounts (or concentrations) in line with the actual reactions.

Still, space is not represented there in a continuous fashion. In Space $\pi$ John, Ewald, and Uhrmacher 2008 (also: SpacePi), processes are equipped with actual coordinates in a two- or three-dimensional space, direction (i.e. a movement function) and radii to indicate the range of their communication potential. Processes are still able to send and receive messages, but only within the given radius instead of the global space or the whole compartment, ensuring that only entities close to each other can react. The formalism thus has hybrid semantics, combining continuous motion of particles with discrete events like collisions and reactions. While the original description of SpacePi covers only rather simple forms of stochastic and directed motion, Brownian motion can also be covered (Haack, Leye, and Uhrmacher 2010).

Whereas entities in Space $\pi$ are circular or spherical, Shape Calculus (Bartocci et al. 2009) (not to be confused with the shape calculus of Schäfer 2005) was designed particularly with molecules with a more complex 3D structure in mind. The modeled entities are 3D shapes moving in space, communication channels represent potential binding sites, and while a binding reaction yields a new process like in the other calculi, the new process' name is not an abstract one but still contains the original components and the binding site they are linked with. Movement of shapes necessitates the application of sophisticated (and computationally expensive) collision detection techniques as investigated in the field of computer graphics. Cell structures can be represented by explicitly specifying actual membranes as 3D processes like the other molecules, just that they do not move or change under most conditions. Shape Calculus is work in progress, and while there is a semantics definition, there does not seem to be a simulator yet.

\subsection{Others}

Cellular Automata are a discrete, step-wise modeling approach based on a large lattice (grid) of identical cells. A cell and its neighbors current state determines the cell's next state. In one of the basic and most well-known examples, Conway's Game of Life, there are only two possible states (live and dead) and very simple rules, however, complex patterns can result (Gardner 1970). In biology they have been used to describe various phenomena (Materi and Wishart 2007), for example liquid solutions containing enzymes and substrates exhibiting known enzyme kinetics in the simulation (e.g. Michaelis-Menten; Kier et al. 1996) or formation of patterns (Deutsch and Dormann 2004), e.g. in tumor growth (Moreira and Deutsch 2002).

For simulation of intracellular dynamics, a variant called Dynamic Cellular Automata (DCA) has been developed (Wishart et al. 2005), taking in aspects of agent-based modeling. An explicit notion of time is added by defining the actual duration of a time step. Unlike with Cellular Automata, where cell states technically do not move even though they may propagate into one direction, interpreting cell state changes as movements is explicitly allowed. Choosing an appropriately small lattice element size close to that of an average protein $(3 \mathrm{~nm})$ and a time step size so small that molecules do not travel farther than one block at a time, macromolecules (e.g. proteins and RNA) are indeed modeled spatially akin to the original Cellular Automata (and to panel (b) of figure 2). Movement of particles is guided by "randomized, discrete-state jumps" intended to mimic Brownian motion, interactions are triggered by adjacency of appropriate particles (e.g. an enzyme phosphorylating or binding to another at a neighboring cell). Wishart et al. (2005) also add small molecules, of which several can be located inside one cell (unlike macromolecules), and non-mobile "supermolecules" to model membranes (and DNA, interestingly). Using these extensions and their simulator implementation SimCell, they are able to model intracellular phenomena spatially, albeit only on a two-dimensional grid.

The discrete event systems specification DEVS is another modeling approach used for hierarchical (Maus et al. 2008, Uhrmacher et al. 2007) and even for spatial simulation of biological systems (Goldstein and Wainer 2009). However, in the later case the laws of continuous movement as well as the model itself are specified as DEVS and executed by the discrete event simulation machine.

Similarly to DEVS, statecharts (Harel 1987), whose origin lies in software engineering, support a hierarchical modeling of cell biological systems (Holcombe and Bell 1998, Kam, Cohen, and Harel 2002), but in a more visual way. They have even been applied to cover basic spatial phenomena involving the fate of adjacent cells and the interplay with gene regulation within them (Fisher, Piterman, Hubbard, Stern, and Harel 2005). Also similarly to DEVS they do not support an easy modeling of biochemical reactions. Biocharts (Kugler, Larjo, and Harel 2010) are a recent adaptation suited to cell biology. They promise to be a powerful, visual language for describing biological systems. However, especially a definition of their semantics is still work in progress (Harel and Kugler 2010).

\section{COMBINING FEATURES OF SPATIAL MODELING}

The diverse modeling formalisms support different sets of features that are of interest in spatial modeling. It appears a natural next step to explore the combination of features and the implications for the modeling formalisms. 


\section{Bittig and Uhrmacher}

Dynamic Cellular Automata combine, as mentioned, the lattice-based one-(macro-)molecule-per-cell approach of Cellular Automata with the subvolumes with homogeneous particle distribution for micromolecules (figure 2 (b) and (c)). The latter requires the assumption that micromolecules are abundant for stochastic effects to be negligible and that their concentration gradient across distances corresponding to the lattice cell size is small. The discretization of space for macromolecule requires stronger assumptions: that movements can be adequately covered when only allowing steps corresponding to the average molecule size, and that macromolecule sizes are similar enough that no two macromolecules could in principle fit into the area of one lattice cell, and that no macromolecule would actually cover more than one. Aspects of compartmentalization (figure 2 (a)) are also covered by the approach, although compartments are only implicitly defined by the location of immovable membrane proteins. In principle, (static) compartments could also be covered by incorporating the separation of compartments into the lattice element/subvolume borders, treating movement between subvolumes of different compartments unlike movement between subvolumes of the same compartment.

In modeling moving in continuous space, multi-level approaches can be used to bridge the gap between the high computational costs of the physically most realistic simulation approaches (molecular \& Brownian dynamics) and the less realistic approximation of the faster simulation algorithms (e.g. the Next Subvolume Method). For example, to describe the phenomenon of macromolecular crowding (Fulton 1982, Ellis and Minton 2006), Jeschke and Uhrmacher (2008) also consider small abundant particles to be homogeneously distributed within appropriately small subvolumes and simulate only the continuous motion of individual large molecules. They thus have real coordinates and sizes associated with the large molecules, while the location of small molecules is only given by the subvolume they are located in (which have, themselves, boundaries defined by real-valued functions). The small molecules behavior depends only on the subvolume's volume and the amount of other small particles in it, plus appropriate probabilities for diffusion into neighboring subvolumes. Issues that arise here include how to deal with large molecules intersecting several subvolumes, with movement of large molecules into a subvolume pushing out more small molecules into neighboring subvolumes than would otherwise move there, and reactions of large with small molecules. Currently available modeling formalisms are only able to describe some aspects of what is done here, i.e. the combination of panels (c) and (f) of figure 2. SpacePi could cover the continuous movement of the large particles, but lacks representation of their spatial extension. Shape Calculus covers this, but is more focused on binding capabilities of these shapes and too much work in progress to be reasonably evaluated here. $\pi$-calculus based modeling approaches allowing description of subvolumes with homogeneously distributed particles exist in $\mathcal{L} \pi$ and $\pi(\mathcal{L})$. It remains to be seen which one can cover the NSM best and how it can be linked with the continuously moving macroparticles.

Compartments and distribution gradients (figure 2 (a) and (d)) are already combined in the Virtual Cell modeling environment mentioned in section 4.1. The assumptions and abstractions behind this are basically those given for differential equation-based modeling (section 4.1). Thus, in Virtual Cell membranes that separate spatially resolved compartments are treated as discontinuities in the solution of the reaction-diffusion equations and handled differently. Thus, Virtual Cell exploits PDE as part of its models semantics, but not as a modeling formalism.

Finally, instead of individual particles moving through subvolumes of stochastically simulated populations, one could image a combination of individual particles moving "over" a continuously changing concentration of other (smaller) particles, thus again combining stochasticity and determinism (and figure 2 (d) with (f) or (e), for that matter). Handling interactions of micromolecules (population level) with macromolecules (individual level) would be tricky, or at least require the assumption that the actual amount of small molecules represented by the concentration changes only negligibly due to reaction of the small with large ones. Even more challenging will be the representation of the effect of macromolecules on the diffusion of surrounding micromolecules, i.e. the link from the individual level back to the population level. Anyway, since this approach includes PDEs, any modeling formalism for it would likely be more difficult to develop than one for what is discussed in the previous paragraph.

\section{CONCLUSION}

The insight that space plays a crucial role in understanding the dynamics of cell biological systems has fostered the development of diverse modeling approaches during the last years. With these languages the assumption is left behind that molecules are homogeneously distributed in a cell. Each of these approaches offers a specific perception on the system under study and its spatial dimension. The cell is structured into compartments, those structures are subject to dynamic changes, entities move in continuous space, space is structured into lattices, individual molecules or entire species are the key actors exhibiting in some cases a spatial dimension themselves.

The challenge lies in combining different features and doing so in a lean language equipped with a clear semantics. Current applications imply that movement in continuous space and stochastic approaches are a fruitful avenue of research.

\section{ACKNOWLEDGMENTS}

We would like to thank Carsten Maus, Fiete Haack, and Mathias John for fruitful discussion of levels and scales, MJ also for discussion of process calculi, CM also for helpful comments on rule-based modeling and the whole manuscript. 
Figure 1 is composed of Public Domain images (except "interaction networks": own work) found mostly via wikimedia commons <commons.wikimedia.org/wiki/Main_Page>. This research is supported by the German Research Foundation (DFG) within the context of the project DIEM OSIRIS (Integrative Development of Modeling and SImulation Methods for RegeneratIve Systems).

\section{REFERENCES}

Adcock, S. A., and J. A. McCammon. 2006, May. Molecular dynamics: Survey of methods for simulating the activity of proteins. Chemical Reviews 106 (5): 1589-1615.

Ahuatzi, D., A. Riera, R. Peláez, P. Herrero, and F. Moreno. 2007, February. Hxk2 regulates the phosphorylation state of mig1 and therefore its nucleocytoplasmic distribution. Journal of Biological Chemistry 282 (7): 4485-4493.

Alber, M. S., M. A. Kiskowski, J. A. Glazier, and Y. Jiang. 2002. On cellular automaton approaches to modeling biological cells. In In IMA Mathematical Systems Theory in Biology, Communication, and Finance, Volume 134, $1-39$.

Bardwell, L., X. Zou, Q. Nie, and N. L. Komarova. 2007, May. Mathematical models of specificity in cell signaling. Biophysical journal 92 (10): 3425-3441.

Bartocci, E., F. Corradini, M. R. Di Berardini, E. Merelli, and L. Tesei. 2009. Shape calculus - a spatial calculus for 3D colliding shapes. Technical report, School of Sciences and Technology, University of Camerino, Via Madonna delle Carceri 9, 62032, Camerino (MC), Italy.

Bartocci, E., F. Corradini, M. R. Di Berardini, E. Merelli, and L. Tesei. 2009. A shape calculus for biological processes. In 11th Italian Conference on Theoretical Computer Science, 30-33.

Blinov, M. L., J. R. Faeder, B. Goldstein, and W. S. Hlavacek. 2004, November. Bionetgen: software for rule-based modeling of signal transduction based on the interactions of molecular domains. Bioinformatics (Oxford, England) 20 (17): 3289-3291.

Brandt, A. 2001, December. Multiscale scientific computation: Review 2001. In Multiscale and Multiresolution Methods: Theory and Applications (Lecture Notes in Computational Science and Engineering), ed. T. J. Barth, T. F. Chan, and R. Haimes, 1-96. Springer.

Brodo, L., P. Degano, and C. Priami. 2007. A stochastic semantics for bioambients. In Parallel Computing Technologies, ed. V. Malyshkin, Volume 4671 of Lecture Notes in Computer Science, Chapter 3, 22-34. Berlin, Heidelberg: Springer Berlin Heidelberg.

Cardelli, L. 2005. Brane calculi. In Computational Methods in Systems Biology, 257-278.

Cardelli, L., and A. D. Gordon. 2000, June. Mobile ambients. Theoretical Computer Science 240 (1): 177-213.

Ciocchetta, F., and M. Guerriero. 2009, January. Modelling biological compartments in bio-PEPA. Electronic Notes in Theoretical Computer Science 227:77-95.

Ciocchetta, F., and J. Hillston. 2008, January. Bio-PEPA: An extension of the process algebra PEPA for biochemical networks. Electronic Notes in Theoretical Computer Science 194 (3): 103-117.

Danos, V., J. Feret, W. Fontana, R. Harmer, and J. Krivine. 2007. Rule-based modelling of cellular signalling. In Proceedings of the 18 th International Conference on Concurrency Theory (CONCUR'07), Lecture Notes in Computer Science, Volume 4703, 17-41.

Danos, V., J. Feret, W. Fontana, R. Harmer, and J. Krivine. 2009. Rule-based modelling and model perturbation. In Transactions on Computational Systems Biology XI, ed. C. Priami, R.-J. Back, and I. Petre, Volume 5750, Chapter 6, 116-137. Berlin, Heidelberg: Springer Berlin Heidelberg.

Degano, P., D. Prandi, C. Priami, and P. Quaglia. 2006, October. Beta-binders for biological quantitative experiments. Electronic Notes in Theoretical Computer Science 164 (3): 101-117.

Degenring, D., M. Röhl, and A. M. Uhrmacher. 2004, July. Discrete event, multi-level simulation of metabolite channeling. Biosystems 75 (1-3): 29-41.

Deutsch, A., and S. Dormann. 2004, October. Cellular automaton modeling of biological pattern formation. 1 ed. Birkhäuser Boston.

Dijkstra, E. W. 1982. On the role of scientific thought. In Selected Writings on Computing: A Personal Perspective, 60-66. Springer-Verlag.

Dolbow, J., M. A. Khaleel, and J. Mitchell. 2004, December. Multiscale mathematics initiative: A roadmap. Technical report, Department of Energy, USA.

Dumont, J. E., F. Pécasse, and C. Maenhaut. 2001, July. Crosstalk and specificity in signalling are we crosstalking ourselves into general confusion? Cellular Signalling 13 (7): 457-463.

Elf, J., A. Doncic, and M. Ehrenberg. 2003, July. Mesoscopic reaction-diffusion in intracellular signaling. In Fluctuations and Noise in Biological, Biophysical, and Biomedical Systems, ed. S. M. Bezrukov, H. Frauenfelder, and F. Moss, Volume 5110 of Proc. SPIE, 114-124. Society of Photo-Optical Instrumentation Engineers (SPIE): SPIE.

Elf, J., and M. Ehrenberg. 2004, December. Spontaneous separation of bi-stable biochemical systems into spatial domains of opposite phases. Systems biology 1 (2): 230-236. 
Ellis, R. J., and A. P. Minton. 2006, May. Protein aggregation in crowded environments. Biological Chemistry 387 (5): 485-497.

Ermak, D. L., and J. A. McCammon. 1978. Brownian dynamics with hydrodynamic interactions. The Journal of Chemical Physics 69 (4): 1352-1360.

Fisher, J., N. Piterman, Hubbard, M. J. Stern, and D. Harel. 2005, February. Computational insights into caenorhabditis elegans vulval development. Proceedings of the National Academy of Sciences of the United States of America 102 (6): 1951-1956.

Fulton, A. 1982, September. How crowded is the cytoplasm? Cell 30 (2): 345-347.

Funahashi, A., Y. Matsuoka, A. Jouraku, M. Morohashi, N. Kikuchi, and H. Kitano. 2008, July. Celldesigner 3.5: A versatile modeling tool for biochemical networks. Proceedings of the IEEE 96 (8): 1254-1265.

Galpin, V. 2010. Continuous approximation of PEPA models and Petri nets. International Journal of Computer Aided Engineering and Technology 2:324-339.

Gardner, M. 1970. Mathematical games: The fantastic combinations of John Conway's new solitaire game 'Life'. Scientific American 223 (4): 120-123.

Gillespie, D. T. 1976, December. A general method for numerically simulating the stochastic time evolution of coupled chemical reactions. Journal of Computational Physics 22 (4): 403-434.

Goldstein, R., and G. Wainer. 2009, December. DEVS-based design of spatial simulations of biological systems. In Proceedings of the 2009 Winter Simulation Conference, ed. M. D. Rossetti, R. R. Hill, B. Johansson, A. Dunkin, and R. G. Ingalls, 743-754: IEEE Computer Science.

Haack, F., S. Leye, and A. M. Uhrmacher. 2010, April. A flexible architecture for modeling and simulation of diffusional association. In From Biology to Concurrency and back, ed. E. Merelli and P. Quaglia, 70-84.

Harel, D. 1987, June. Statecharts: A visual formalism for complex systems. Science of Computer Programming 8 (3): 231-274.

Harel, D., and H. Kugler. 2010, July. Some thoughts on the semantics of biocharts, 185-194. Springer-Verlag Berlin Heidelberg.

Harris, L. A., J. S. Hogg, and J. R. Faeder. 2009, December. Compartmental rule-based modeling of biochemical systems. In Proceedings of the 2009 Winter Simulation Conference, ed. M. D. Rossetti, R. R. Hill, B. Johansson, A. Dunkin, and R. G. Ingalls, 908-919: IEEE Computer Science.

Haugh, J. M. 2002, February. A unified model for signal transduction reactions in cellular membranes. Biophysical journal 82 (2): 591-604.

Heyes, D. M., J. Baxter, U. Tüzün, and R. S. Qin. 2004, September. Discreteelement method simulations: from micro to macro scales. Philosophical Transactions of the Royal Society of London. Series A: Mathematical, Physical and Engineering Sciences 362 (1822): 1853-1865.

Hlavacek, W. S., J. R. Faeder, M. L. Blinov, R. G. Posner, M. Hucka, and W. Fontana. 2006, July. Rules for modeling signal-transduction systems. Sci. STKE 2006 (344): re6+.

Holcombe, M., and A. Bell. 1998. Computational models of immunological pathways. In IPCAT '97: Proceedings of the second international workshop on Information processing in cell and tissues, 213-226. New York, NY, USA: Plenum Press.

Hoops, S., S. Sahle, R. Gauges, C. Lee, J. Pahle, N. Simus, M. Singhal, L. Xu, P. Mendes, and U. Kummer. 2006, December. Copasi-a complex pathway simulator. Bioinformatics 22 (24): 3067-3074.

Hucka, M., A. Finney, H. M. Sauro, H. Bolouri, J. C. Doyle, H. Kitano et al. 2003, March. The systems biology markup language (SBML): a medium for representation and exchange of biochemical network models. Bioinformatics 19 (4): 524-531.

Jeschke, M., and A. M. Uhrmacher. 2008, December. Multi-resolution spatial simulation for molecular crowding. In Proceedings of the 2008 Winter Simulation Conference, ed. S. J. Mason, R. R. Hill, L. Moench, and O. Rose, 1384-1392: IEEE.

John, M., R. Ewald, and A. M. Uhrmacher. 2008, January. A spatial extension to the $\pi$ calculus. Electronic Notes in Theoretical Computer Science 194 (3): 133-148.

John, M., C. Lhoussaine, and J. Niehren. 2009. Dynamic compartments in the imperative $\pi$-calculus. In Computational Methods in Systems Biology, ed. P. Degano and R. Gorrieri, Volume 5688, Chapter 16, 235-250. Berlin, Heidelberg: Springer Berlin Heidelberg.

John, M., C. Lhoussaine, J. Niehren, and A. Uhrmacher. 2010. The attributed pi-calculus with priorities. In Transactions on Computational Systems Biology XII, ed. C. Priami, R. Breitling, D. Gilbert, M. Heiner, and A. M. Uhrmacher, Volume 5945, Chapter 2, 13-76. Berlin, Heidelberg: Springer Berlin Heidelberg.

Kam, N., I. R. Cohen, and D. Harel. 2002. Modeling biological reactivity: statecharts vs. boolean logic. In AVI 'O2: Proceedings of the Working Conference on Advanced Visual Interfaces, 345-353. New York, NY, USA: ACM.

Kholodenko, B. N. 2006, February. Cell-signalling dynamics in time and space. Nature Reviews Molecular Cell Biology 7 (3): $165-176$.

Kier, L. B., C. K. Cheng, B. Testa, and P.-A. Carrupt. 1996, August. A cellular automata model of enzyme kinetics. Journal of Molecular Graphics 14 (4): 227-231. 
Kugler, H., A. Larjo, and D. Harel. 2010, July. Biocharts: a visual formalism for complex biological systems. Journal of the Royal Society, Interface / the Royal Society 7 (48): 1015-1024.

Lai, X., S. Nikolov, O. Wolkenhauer, and J. Vera. 2009, August. A multi-level model accounting for the effects of jak2-stat5 signal modulation in erythropoiesis. Computational Biology and Chemistry 33 (4): 312-324.

Li, J., W. Ge, J. Zhang, and M. Kwauk. 2005, June. Multi-scale compromise and multi-level correlation in complex systems. Chemical Engineering Research and Design 83 (6): 574-582.

Materi, W., and D. S. Wishart. 2007, April. Computational systems biology in drug discovery and development: methods and applications. Drug Discovery Today 12 (7-8): 295-303.

Maus, C. 2008. Component-based modelling of rna structure folding. In Computational Methods in Systems Biology, ed. M. Heiner and A. M. Uhrmacher, Volume 5307, Chapter 8, 44-62. Berlin, Heidelberg: Springer Berlin Heidelberg.

Maus, C., M. John, M. Röhl, and A. Uhrmacher. 2008. Hierarchical modeling for computational biology. In Formal Methods for Computational Systems Biology, ed. M. Bernardo, P. Degano, and G. Zavattaro, Volume 5016 of Lecture Notes in Computer Science, Chapter 4, 81-124. Berlin, Heidelberg: Springer Berlin Heidelberg.

Mazemondet, O., M. John, C. Maus, A. M. Uhrmacher, and A. Rolfs. 2009, December. Integrating diverse reaction types into stochastic models - a signaling pathway case study in the imperative pi-calculus. In Proceedings of the 2009 Winter Simulation Conference, ed. M. D. Rossetti, R. R. Hill, B. Johansson, A. Dunkin, and R. G. Ingalls, 932-943: IEEE Computer Science.

Milner, R. 1999, June. Communicating and mobile systems: the pi-calculus. Cambridge University Press.

Möhring, M. 1996. Social science multilevel simulation with mimose. In Social Science Microsimulation, ed. K. G. Troizsch and Others, 282-306. Springer-Verlag Berlin.

Moreira, J., and A. Deutsch. 2002. Cellular automaton models of tumor development: A critical review. Advances in Complex Systems (ACS) 5 (02): 247-267.

Northrup, S. H., S. A. Allison, and J. A. McCammon. 1984. Brownian dynamics simulation of diffusion-influenced bimolecular reactions. The Journal of Chemical Physics 80 (4): 1517-1524.

Pedersen, M., and G. Plotkin. 2010. A language for biochemical systems: Design and formal specification. In Transactions on Computational Systems Biology XII, ed. C. Priami, R. Breitling, D. Gilbert, M. Heiner, and A. M. Uhrmacher, Volume 5945, Chapter 3, 77-145. Berlin, Heidelberg: Springer Berlin Heidelberg.

Priami, C. 1995, July. Stochastic $\pi$-calculus. The Computer Journal 38 (7): 578-589.

Priami, C., and P. Quaglia. 2005, April. Beta binders for biological interactions. In Computational Methods in Systems Biology, 20-33. Springer Berlin Heidelberg.

Recalde, L., S. Haddad, and M. Silva. 2007. Continuous petri nets: Expressive power and decidability issues. In Automated Technology for Verification and Analysis, ed. K. S. Namjoshi, T. Yoneda, T. Higashino, and Y. Okamura, Volume 4762 of Lecture Notes in Computer Science, Chapter 26, 362-377. Berlin, Heidelberg: Springer Berlin Heidelberg.

Regev, A., E. M. Panina, W. Silverman, L. Cardelli, and E. Shapiro. 2004, September. Bioambients: an abstraction for biological compartments. Theoretical Computer Science 325 (1): 141-167.

Regev, A., and E. Shapiro. 2004. The $\pi$-calculus as an abstraction for biomolecular systems. In Modelling in Molecular Biology (Natural Computing Series), ed. G. Ciobanu and G. Rozenberg, 219-266. Springer.

Schäfer, A. 2005. A calculus for shapes in time and space. In Theoretical Aspects of Computing - ICTAC 2004, 463-477. Springer-Verlag Berlin Heidelberg.

Schaff, J., C. C. Fink, B. Slepchenko, J. H. Carson, and L. M. Loew. 1997, September. A general computational framework for modeling cellular structure and function. Biophysical Journal 73 (3): 1135-1146.

Schmidt, H., and M. Jirstrand. 2006, February. Systems biology toolbox for matlab: a computational platform for research in systems biology. Bioinformatics 22 (4): 514-515.

Shimizu, T. S., S. V. Aksenov, and D. Bray. 2003, May. A spatially extended stochastic model of the bacterial chemotaxis signalling pathway. Journal of Molecular Biology 329 (2): 291-309.

Slepchenko, B. M., J. C. Schaff, I. Macara, and L. M. Loew. 2003, November. Quantitative cell biology with the virtual cell. Trends in cell biology 13 (11): 570-576.

Stefanek, A., M. Vigliotti, and J. T. Bradley. 2009, August. Spatial extension of stochastic pi calculus. In 8th Workshop on Process Algebra and Stochastically Timed Activities, 109-117.

Stundzia, A. B., and C. J. Lumsden. 1996, August. Stochastic simulation of coupled reaction-diffusion processes. Journal of Computational Physics 127 (1): 196-207.

Takahashi, K., S. Arjunan, and M. Tomita. 2005, March. Space in systems biology of signaling pathways - towards intracellular molecular crowding in silico. FEBS Letters 579 (8): 1783-1788.

Takahashi, K., K. Kaizu, B. Hu, and M. Tomita. 2004, March. A multi-algorithm, multi-timescale method for cell simulation. Bioinformatics 20 (4): 538-546.

Tilly, C. 1997. Micro, macro, or megrim? Columbia University August 1997 (August).

Uhrmacher, A. M., R. Ewald, M. John, C. Maus, M. Jeschke, and S. Biermann. 2007, December. Combining micro and macro-modeling in DEVS for computational biology. In Proceedings of the 2007 Winter Simulation Conference, 
ed. S. G. Henderson, B. Biller, M. H. Hsieh, J. Shortle, J. D. Tew, and R. R. Barton, 871-880. Piscataway, NJ, USA: IEEE Press.

van Zon, J. S., and P. R. ten Wolde. 2005, April. Simulating biochemical networks at the particle level and in time and space: Green's function reaction dynamics. Physical Review Letters 94 (12): 128103+.

Weinan, E., and B. Engquist. 2003. Multiscale modeling and computation. Notices Amer. Math. Soc 50 (50): 1062-1070.

Wishart, D. S., R. Yang, D. Arndt, P. Tang, and J. Cruz. 2005, January. Dynamic cellular automata: An alternative approach to cellular simulation. In Silico Biology 5 (2): 139-161.

Yang, L., and P. A. Iglesias. 2009. Modeling spatial and temporal dynamics of chemotactic networks. Methods in molecular biology (Clifton, N.J.) 571:489-505.

Zeigler, B. P., T. G. Kim, and H. Praehofer. 2000. Theory of modeling and simulation. Orlando, FL, USA: Academic Press, Inc.

\section{AUTHOR BIOGRAPHIES}

ARNE T. BITTIG holds a Diplom degree in Computer Science from the University of Rostock. He is currently a PhD student in the DFG Research Training School dIEM oSiRiS and a member of the modeling and simulation group at the University of Rostock. His main research interest is continuous space in the modeling of biological systems. His email address is <arne.bittig@uni-rostock.de>.

ADELINDE M. UHRMACHER is a Professor at the Department of Computer Science at the University of Rostock and head of the Modeling and Simulation Group. Her research interests are in modeling and simulation methodologies and their applications. Her e-mail address is <lin@informatik.uni-rostock.de> and her web page is $<$ www. informatik. uni-rostock. de/ ${ }^{\sim}$ lins. 